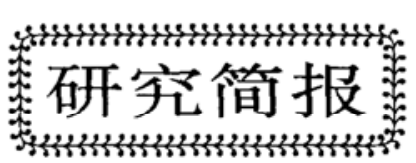

\title{
开放逻辑中的 $\boldsymbol{R}$-重构
}

\author{
苏开乐
}

(南京大学数学系, 南京 210008)

关篗词 $R$ - 重构、假设、事实反驱

\section{1 引言及基本概念}

为了刻画知识的增长, 更新以及假说的进化, $\mathrm{Li}^{[1]}$ 最近建立了一个开放的逻辑理论. 开 放逻辑的一个基本概念就是 $R$-重构, $R$-重构不具有唯一性, 文献[1] 中指出了这一点. 对任 意给定的假说 $\Gamma$ 和语句 $A$ 使得 $\Gamma=A$ 且 $A$ 有事实反驭, 我们给出了 $\Gamma$ 关于 $A$ 的事实反玫的 $R-$ 重构唯一的充要条件. 令 $R$-recons $(\Gamma, A)$ 为所有 $\Gamma$ 关于 $A$ 的事实反驳的 $R$-重构的集合, 我 们讨论了当 $\Gamma, A$ 变化时, $R$-recons $(\Gamma, A)$ 的基数的可能变化范围.

我们采用通常一阶逻辑语言 $L$, 一假说就是一相容的句子的集合. 我们采用的基本概念 都与文献[1] 中相同. 令 $\Gamma \models A$ 且 $A$ 受到事实反驳, 根据文献[1] 中的定理 2.2 和定理 2.3 我们 有对任何句子集 $S$.

(1) $S \in R$-recons $(\Gamma, A)$ 当且仅当存在某个句子集 $Q$ 使得 $S=Q \bigcup\{\urcorner A\}$ 且 $Q$ 是与 $\urcorner A$ 相容 的 $\Gamma$ 的极大子集.

在本文为方便起见约定当 $\Gamma \not \neq A$ 时 $R$-recons $(\Gamma, A)=\{\Gamma\} ; A$ 不受到事实反驳即 $A$ 永真 时, $R$-recons $(\Gamma, A)=\varnothing$. 即对任何假说 $\Gamma$ 和句子 $A$ 我们承认 (1) 式.

\section{2 主 要 结 果}

引理 1 对任何假说 $\Gamma$, 句子 $X$ 和 $S \subseteq \Gamma$, 如果 $S$ 与 $X X$ 相容, 那么存在句子集 $Q \subseteq \Gamma$, 使 得 $S \subseteq Q$ 且 $Q$ 是与 $\urcorner X$ 相容的 $\Gamma$ 的极大子集, 即

证应用Zorn 引理及一阶逻辑的紧性定理.

$$
Q \bigcup\{7 X\} \in R \text {-recons }(\Gamma, X) \text {. }
$$

定理 1 对任何假说 $\Gamma$ 及句子 $X, R$-recons $(\Gamma, X)$ 的基数小于或等于 1 当且仅当对任 何 $X_{1}, \cdots, X_{m} \in \Gamma$ 若 $X_{1} \& \cdots \& X_{m} \models X$ 则存在 $0<n \leqslant m$ 使得 $X_{n} \vDash X$

证 “ $\Rightarrow$ ” 部分. 假设 $R$-recons $(\Gamma, X)$ 的基数小于或等于 1 , 又设 $X_{1}, \cdots, X_{m} \in \Gamma, X_{1} \&$ $\cdots \& X_{m} \vDash X$. 若 $X_{1} \not \neq X$ 则由引理 $1\left\{X_{1}\right\}$ 可扩充为一个与 $7 X$ 相容的 $\Gamma$ 的极大子集 $S$, 即 $S \cup$ $\{\neg X\} \in R$-recons $(\Gamma, X)$. 由 $X_{1} \& \cdots \& X_{m} \mid=X$ 知存在 $X_{n}, 0<n \leqslant m$ 使得 $X_{n} \notin S$ 否则 $S$ 将与 $7 X$ 不相 容. 又若 $X_{n} \not X$, 则同样由引理 2 存在 $S^{1}$ 使得 $X_{n} \in S^{1}, S^{1} \subset \Gamma, S^{1} \cup\{7 X\} \in R$-recons $(\Gamma, X)$. 由

1993-04-05 收稿. 
此可以断言 $X_{1} \models X$ 或者 $X_{n} \models X$, 否则 $R$-recons $(\Gamma, X)$ 中将有两个句子集即 $\left.S \cup\{\urcorner X\right\}$ 和 $S^{\prime}$ $\bigcup\{7 X\}$, 而 $\mathrm{S} \cup\{7 X\} \neq S^{\prime} \cup\{7 X\}$ 因为 $X_{n}$ 属于右边, 且不属于左边 $\left(X_{n} \notin S\right.$ 且 $\left.X_{n} \not \neq\right\urcorner X$ 否则 $S$ $\cup\left\{X_{n}\right\}$ 与 $7 X$ 相容, 由 $S$ 的极大性 $\left.X_{n} \in S\right)$. 从而 $R$-recons $(\Gamma, X)$ 的基数至少为 2 , 与假设矛 盾.

“Æ”部分. 设 $R$-recons $(\Gamma, X)$ 的基数大于 1 即有 $S, S^{\prime}$ 使得 $S \cup\{7 X\} \in R$-recons $(\Gamma, X)$, $S^{\prime} \cup\{7 X\} \in R$-recons $\left.(\Gamma\urcorner X,\right)$, 且 $S, S^{\prime}$ 是与 $\urcorner X$ 相容的 $\Gamma$ 的极大子集, 且 $S^{\prime} \neq S$. 由 $S^{\prime} \neq S$ 知有 $X_{0} \in S$ 使得 $X_{0} \notin S^{\prime}$, 从而由 $S^{\prime}$ 的极大性, 有 $X_{1}, \cdots, X_{m} \in S^{\prime}\left\{X_{0}, \cdots, X_{m}\right\}$ 与 $\urcorner X$ 不相容, 即 $X_{0} \& X_{1}$ $\& \cdots \& X_{m} \vDash X$ 但 $X_{0} \not \neq X$ 因为 $X_{0} \in S$ 从而 $X_{0}$ 与 $\urcorner X$ 相容; 同样因为 $X_{1}, \cdots, X_{m} \in S^{\prime}$ 我们有 $X_{i} \mid \neq X$, $0<i \leqslant m$. 综合以上, 假设 $R$-recons $(\Gamma, X)$ 的基数大于 1 , 我们有 $X_{0}, \cdots, X_{m} \in \Gamma$ 使得 $X_{0} \& X_{1} \& \cdots \& X_{m} \models X$ 但对任何 $0 \leqslant i \leqslant m$ 都有 $X_{i} \not \neq X_{0}$ “部分得证.

引理 2 设 $\Gamma_{0}, \Gamma_{1}$ 是两假说使得 $\Gamma_{0} \cap \Gamma_{1}=\varnothing$ 且对任何 $T \subseteq \Gamma_{0}$ 若 $T \cup \Gamma_{1} \vDash X$ 则 $T \models X$, 即么对任何句子集 $S$,

(i) $S \in R$-recons $\left(\Gamma_{0}, X\right)$ 当且仅当 $S \bigcup \Gamma_{1} \in R$-recons $\left(\Gamma_{0} \cup \Gamma_{1}, X\right)$.

(ii) 对任何 $Q \in R\left(\Gamma_{0} \cup \Gamma_{1}, X\right)$ 存在唯一的 $S \in R$-recons $\left(\Gamma_{0}, X\right)$ 使得 $Q=S \cup \Gamma_{1}$.

(iii) $R$-recons $\left(\Gamma_{0} \cup \Gamma_{1}, X\right)$ 与 $R$-recons $\left(\Gamma_{0}, X\right)$ 的基数相等.

证 (iii) 直接由 (i), (ii) 得到, (i), (ii) 由 $R$ - 重构的定义即 (1) 式和引理中的条件得 到.

引理 3 设 $\Gamma_{0}, \Gamma_{1}$ 是两假说, $X$ 是语句使得 (i) $\Gamma_{1}$ 中的句子与 $\Gamma_{0}$ 中的句子以及 $X$ 没含 公共的初始谓词, 函项和常元. (ii) $\Gamma_{1}$ 有无穷可数模型. (iii) 对任何 $T_{1}, \cdots, T_{m} \in \Gamma_{0}$ 若 $T_{1} \& \cdots$ $\cdots \& T_{m} \rightarrow X$ 在任何无穷可数模型中真则 $T_{1} \& \cdots \& T_{m} \rightarrow X$ 永真. 那么对任何 $T \subseteq \Gamma_{0}$ 若 $T \cup \Gamma_{1}$ $\models X$ 则 $T \models X$ 成立.

证应用一阶逻辑中 Criag 插值定理, 并注意到任何不含有初始谓词、函词, 常元的公 式如果在某个可数模型中真则在任何可数模型中真.

定理 2 在含有无穷可数多个常元和无穷可数多个一元谓词作为初始符号的一阶逻辑 语言中, 存在假说 $\Gamma$ 和语句 $X^{0}, \cdots, X^{n}, \cdots ; X^{\omega}, X^{*}$ 使得

（i）对任何自然数 $n, R$-recons $\left(\Gamma, X^{n}\right)$ 的基数为 $n$.

(ii) $R$-recons $\left(\Gamma, X^{\omega}\right)$ 的基数为 $\boldsymbol{\aleph}_{0}$ (无穷可数).

(iii) $R$-recons $\left(\Gamma, X^{*}\right)$ 的基数为 $2^{\mathrm{K}_{0}}$ (连续统).

证 令 $p^{1}, p^{2}, \cdots, p^{n}, \cdots ; p^{\omega} ; p_{1}{ }^{*}, p_{2}{ }^{*}$ 为一元谓词初始符号, 对任何自然数 $m, C_{m}^{1}$, $C_{m}^{2}, \cdots, C_{m}^{n}, \cdots, C_{m}^{\omega}, C_{m}^{*}$ 为两两不同的常元. 我们令

$$
\Gamma_{0}=\varnothing, X^{0} \text { 为一永真句子如 } \forall x(x=x) \text {. }
$$

对一切自然数 $n>0, \Gamma_{n}=\left\{A_{j}^{n}\right\}_{j<n}$, 其中 $A_{j}^{n}$ 为 $\forall x\left(x \neq C_{j}^{n} \rightarrow p^{n}(x)\right) \& C_{0}^{n} \neq C_{j}^{n} \& \cdots \& C_{j-1}^{n} \neq C_{j}^{n}$; $X^{n}$ 为 $\forall x p^{n}(x)$.

$\Gamma_{\omega}=\left\{A_{j}^{\omega}\right\}_{j}$ 为自然数, 其中 $A_{j}^{\omega}$ 为

$$
\begin{gathered}
\forall x\left(x \neq C_{j}^{\omega} \rightarrow p^{\omega}(x)\right) \& C_{0}^{\omega} \neq C_{j}^{\omega} \& \cdots \& C_{j-1}^{\omega} \neq C_{j}^{\omega} ; X^{\omega} \text { 为 } \forall x p^{\omega}(x), \\
\Gamma_{*}=\left\{P_{1}{ }^{*}\left(C_{0}{ }^{*}\right), P_{2}{ }^{*}\left(C_{0}{ }^{*}\right), \cdots, P_{1}{ }^{*}\left(C_{n}{ }^{*}\right), P_{2}{ }^{*}\left(C_{n}{ }^{*}\right), \cdots\right\},
\end{gathered}
$$

$X^{*}$ 为 $\exists x\left(p_{1}^{*}(x) \& p_{2}^{*}(x)\right)$.

最后令 $\Gamma=\left(U\left\{\Gamma_{i}: i \in \omega\right\}\right) \cup \Gamma_{\omega} \cup \Gamma_{*}$. 
我们有 $R$-recons $\left(\Gamma_{1} X^{0}\right)=\varnothing$. 对 $n>0, R$-recons $\left.\left(\Gamma_{n}, X^{n}\right)=\left\{\left\{A_{j}^{n},\right\urcorner X^{n}\right\}: j<n\right\}$ 其基数为 $n$. $R$-recons $\left.\left(\Gamma_{\omega}, X^{\omega}\right)=\left\{\left\{A_{j}^{\omega},\right\urcorner X^{\omega}\right\}: j \in \omega\right\}$, 其基数为 $\boldsymbol{\aleph}_{0}$.

$R$-recons $\left(\Gamma_{*}, X^{*}\right)=\left\{\Gamma_{s} \cup\left\{7 X^{*}\right\}: S \subset \omega\right\}$, 其中 $\Gamma_{S}=\left\{p_{1}{ }^{*}(i): i \in S\right\} \bigcup\left\{p_{2}{ }^{*}(i): i \notin S\right\}$. $R$-recons $\left(\Gamma_{*}, X^{*}\right)$ 的基数为 $2^{\mathrm{N}_{0}}$.

我们用 $\Delta$ 表示 $1,2, \cdots, n, \cdots ; \omega, *$ 等中的任一个由以上我们只要证明 $R$-recons $\left(\Gamma_{\Delta}, X^{\Delta}\right)$ 与 $R$-recons $\left(\Gamma, X^{\Delta}\right)$ 的基数相等. 由引理 2 只需证对任何 $T \subseteq \Gamma_{\Delta}$ 若 $T \cup\left(\Gamma-\Gamma_{\Delta}\right) \vDash X^{\Delta}$ 则 $T \models X^{\Delta}$, 由引理 3 只需证明条件 (i), (ii), (iii) 对 $\Gamma_{\Delta}, \Gamma-\Gamma_{\Delta}, X^{\Delta}$ 成立. (i), (ii) 易证成 立. 至于条件 (iii) 当 $\Delta$ 为 $1,2, \cdots n, \cdots ; \omega$ 中任一个时, 任何 $T_{1}, \cdots, T_{m} \in \Gamma_{\Delta}, T_{1} \& \cdots \& T_{m} \rightarrow$ $X$ 在任何无穷可数模型中真蕴涵 $\left\{T_{1}, \cdots, T_{m}\right\}$ 有两个不同的元素蕴涵 $T_{1} \& \cdots \& T_{m} \rightarrow X$ 永真. 当 $\Delta$ 为 $*$ 号时, 对任何 $T_{1}, \cdots, T_{m} \in \Gamma_{s}, T_{1} \& \cdots \& T_{m} \rightarrow X^{\Delta}$ 在任何无穷可数模型中为真蕴涵 $\left\{T_{1}\right.$, $\left.\cdots, T_{m}\right\}$ 中存在两个元素 $P_{1}^{*}(i), P_{2}^{*}(i)$, 其中 $i$ 为某个自然数, 蕴涵 $T_{1} \& \cdots \& T_{m} \rightarrow X^{\Delta}$ 永真. 从 而引理 3 中条件 (iii) 也成立. 定理得证.

定理 3 对任何假说 $\Gamma$ 和语句 $X$ 如果 $R$-recons $(\Gamma, X)$ 的基数不可数则等于 $2^{\mathrm{K}_{0}}$.

证 由于我们采用的是可数的一阶语言每个句子都对应一个炣德尔编码, 而句子就看 作为自然数的子集. 根据经典的描述集合论结果,任何用 $\Sigma_{1}^{1}$ 型的 (可含参数) 语句定义的自然 数的集合类的基数如果不可数则等于 $2^{\mathrm{N}_{0}}$. 我们只要证明 $R$-recons $(\Gamma, X)$ 是 $\Sigma_{1}^{1}$ 可定义的即 可. 事实上,对任何 $Z$.

相容 $(Z) \& \forall Y \in(\Gamma-Z)(7$ 相容 $(Z \cup\{y\})$.

$$
Z \in R \text {-recons }(\Gamma, X) \Leftrightarrow Z \subseteq \Gamma \cup\{7 X\} \& 7 X \in Z \&
$$

注意到对任何 $S$, 谓词相容 $(S)$ (表示 $S$ 是相容的句子集) 是 $\Pi_{0}^{1}$ 于 $S$ 的, 从而 $Z \in R$-recons $(\Gamma$, $X)$ 是算术于 $\Gamma$ 的. 因而是 $\Sigma_{1}^{1}$ 于 $\Gamma$ 的.

致谢本文是在导师莫绍揆教授和丁德成教授的指导下完成的, 是丁德成教授主持的 国家“863”课题研讨班集体讨论的结果,作者在此一并表示感谢.

\section{参考 文 献}

[1] Li, W., Science in China, Series A, 1993, 36(3): 362-375.

[2] Shoenfield, J. R., Mathematical Logic, Addison-Wesley Publ. Co., London, 1967.

[3] Jech, T., Set Theory, Academic Press, New York, 1978. 